INFLAMMATORY BOWEL DISEASE

\title{
CDP571, a humanised monoclonal antibody to tumour necrosis factor $\alpha$, for moderate to severe Crohn's disease: a randomised, double blind, placebo controlled trial
}

\author{
W J Sandborn, B G Feagan, G Radford-Smith, A Kovacs, R Enns, A Innes, J Patel
}

Gut 2004;53:1485-1493. doi: 10.1136/gut.2003.035253

See end of article for authors' affiliations

Correspondence to: Dr W J Sandborn, Mayo Clinic, 200 First Street SW Rochester, MN 55905, USA; sandborn.william@ mayo.edu

Revised version received 16 March 2004

Accepted for publication 31 March 2004
Background: Targeting tumour necrosis factor $\alpha$ (TNF- $\alpha$ ) has demonstrated efficacy in Crohn's disease. Aim: To evaluate CDP571, a humanised antibody to TNF- $\alpha$, for treating active Crohn's disease. Patients: A total of 396 patients with moderate to severe Crohn's disease.

Methods: In a 28 week, randomised, double blind, placebo controlled trial, patients received intravenous CDP571 $(10 \mathrm{mg} / \mathrm{kg}$ ) or placebo every eight weeks to week 24 . The primary outcome measure was clinical response (a decrease in the Crohn's disease activity index (CDAl) to $\geqslant 100$ points or remission (CDAl score $\leqslant 150$ points)) at week 28 . A secondary outcome measure was clinical response (using the same definition) at week 2 .

Results: Clinical response occurred at week 28 in 80/263 (30.4\%) CDP571 patients and 31/132 (23.5\%) placebo patients $(p=0.102)$. Clinical response at week 2 occurred in $90 / 263(34.2 \%)$ CDP571 patients and $28 / 132(21.2 \%)$ placebo patients $(p=0.011)$. Post hoc exploratory subgroup analysis of 159 patients with baseline $C$ reactive protein (CRP) $\geqslant 10 \mathrm{mg} /$ l demonstrated significant differences between CDP571 and placebo in clinical response rates at weeks 2 (CDP571, 50/101 (49.5\%); placebo, 9/58 (15.5\%); $p<0.001)$ and 28 (CDP571, 29/101 (28.7\%); placebo, 7/58 (12.1\%); $p=0.018)$. Adverse events occurred at similar frequencies in both treatment groups.

Conclusions: CDP571 is modestly effective for short but not long term treatment of unselected patients with moderate to severe Crohn's disease. The clinical relevance of this short term effect is unclear. Post hoc analysis suggests both short and long term efficacy of CDP571 in patients with elevated baseline CRP $(\geqslant 10 \mathrm{mg} / \mathrm{l})$. CDP571 is well tolerated.
$\mathrm{T}$ he chimeric monoclonal antibody to tumour necrosis factor $\alpha$ (TNF- $\alpha)$, infliximab, is effective for both the short and long term treatment of patients with moderate to severe Crohn's disease and patients with draining enterocutaneous fistulae. ${ }^{1-5}$ However, infliximab is immunogenic and intermittent administration results in human antichimeric antibodies (HACAs) that lead to infusion reactions, loss of efficacy, and delayed hypersensitivity reactions. $^{6-8}$

The process of "humanisation" of monoclonal antibodies entails transfer of the antigen binding regions (complementarity determining regions (CDR)) of the murine variable domain to a human antibody. ${ }^{9}$ The resulting humanised monoclonal antibody is approximately $95 \%$ human protein and is relatively less immunogenic than chimeric antibodies. A humanised monoclonal antibody to human TNF- $\alpha$, CDP571, was constructed by linking the CDR of a murine antihuman TNF monoclonal antibody to a human IgG4 antibody. ${ }^{10}$ Three phase II trials suggested that intravenous CDP571 may be well tolerated and effective for the short term treatment of mild to moderate Crohn's disease and steroid dependent Crohn's disease. ${ }^{11-13}$ The optimal dosing regimen appeared to be $10 \mathrm{mg} / \mathrm{kg}$ every eight weeks. We conducted a 28 week phase III trial in which patients with moderate to severe Crohn's disease received intravenous CDP571 10 mg/kg or placebo every eight weeks through to week 24 .

\section{METHODS}

Selection of patients

Eligible patients, who were at least 18 years of age and had moderate to severe Crohn's disease as defined by a score of 220-450 (inclusive) on the Crohn's disease activity index
(CDAI),${ }^{14}$ took part in the trial between 18 January 2001 and 15 May 2002. The diagnosis of Crohn's disease had been made previously based on radiological, endoscopic, or histological evidence.

Patients with any of the following were not eligible for entry into the trial: infection of a fistula (abscess) (performance of a pelvic magnetic resonance imaging scan for evaluation of an abscess was not mandatory but was at the discretion of the investigator); ulcerative colitis; bowel perforation or evidence of non-inflammatory obstruction within the six months prior to study entry (fixed stenosis at endoscopy or radiography (performance of these diagnostic evaluations was not mandatory but was at the discretion of the investigator)); obstructive symptoms due to significant mechanical obstruction within the three months prior to study entry; small bowel resection $>100 \mathrm{~cm}$ and/or more than the right colon resected; a functional colostomy; or an ileostomy. Patients were also not eligible if they had: a current infection with enteric pathogens; any serious intercurrent infection or other clinically important active disease (such as renal or hepatic disease) within the three months prior to study entry; current or previous malignancy (other than carcinoma of the cervix or basal cell carcinoma successfully treated $>5$ years prior to study entry); current or previous bowel dysplasia within the five years prior to study screening (surveillance colonoscopy was not mandatory but was at the discretion of the investigator); or clinically

Abbreviations: CDAl, Crohn's disease activity index; CDR, complementarity determining region; $\mathrm{CRP}, \mathrm{C}$ reactive protein; $\mathrm{HACA}$ human antichimeric antibody; IBDQ, inflammatory bowel disease questionnaire; ITT, intention to treat; TNF- $\alpha$, tumour necrosis factor $\alpha$ 
important allergies or multiple drug allergies. In addition, drug or alcohol abuse, significant abnormal haematology or biochemical values at study entry, or a history of or concurrent tuberculosis, hepatitis, or human immunodeficiency virus excluded patients from entry into the trial. Pregnant and lactating women were ineligible, as were patients who were uncooperative or unable to comply with study procedures. The institutional review board at each centre approved the study and all participants gave written informed consent.

\section{Concomitant medications}

Patients receiving prednisone/prednisolone for $\geqslant 4$ weeks at a stable dose (maximum allowed dose $20 \mathrm{mg} /$ day for $\geqslant 2$ weeks) were eligible for entry into the trial. Similarly, patients receiving budesonide for $\geqslant 4$ weeks at a stable dose (maximum allowed dose $6 \mathrm{mg}$ ) for $\geqslant 2$ weeks could also be enrolled into the study. Patients treated with parenteral corticosteroids or corticotrophin within four weeks and those in whom corticosteroid treatment was discontinued within two weeks were not eligible. Treatment with azathioprine or 6-mercaptopurine at a stable dose for $\geqslant 16$ weeks, or methotrexate at a stable dose for 12 weeks, was permitted. Patients who discontinued these agents within the four weeks prior to study entry were ineligible. Receipt of sulphasalazine, mesalamine, or balsalazide at a stable dose for $\geqslant 12$ weeks was permitted, as was antibiotic treatment for Crohn's disease at a stable dose for $\geqslant 8$ weeks (provided that the patient agreed to continue the antibiotic during screening and throughout the 28 week trial). Patients who had received sodium cromoglycate, mycophenolate, or cyclosporin within four weeks of study commencement were excluded, as were those who had been treated with more than four doses of opioid containing analgesics or non-steroidal anti-inflammatory drugs (including cyclooxygenase 1 and 2 inhibitors) within two weeks. Taking certain opioids (loperamide, diphenoxylate, codeine) for the control of diarrhoea was permitted.

Patients who had previously participated in a clinical trial of CDP571 or who had been treated with another biological
anti-TNF- $\alpha$ therapy within 12 weeks of study entry were excluded. Patients who had received previous anti-TNF- $\alpha$ therapy and subsequently experienced either a lack of response to the first dose or an infusion reaction that was suspected or confirmed to be associated with an immune response (for example, presence of HACA) were ineligible. Other exclusion criteria included participation in a clinical trial involving an antibody, cytokine, or other immunomodulatory therapy within the three months prior to study entry and taking part in any other clinical trial within one month prior to study entry.

\section{Study medication}

Pharmacists at each centre prepared the intravenous infusions (CDP571 or placebo). Placebo was a 5\% weight/volume solution of dextrose. CDP57l at a dose of $10 \mathrm{mg} / \mathrm{kg}$ body weight or placebo was administered to patients as a two hour intravenous infusion using a $0.2 \mu \mathrm{m}$ filter. Both CDP571 and placebo had a similar clear and colourless appearance. Patients received infusions every eight weeks through to week 24.

\section{Design of the study}

This randomised, double blind, placebo controlled trial was performed at 68 centres in Australia, Bulgaria, Canada, Czech Republic, Hungary, Poland, and the USA (see appendix). The randomisation procedure was stratified according to the use of the following concomitant medications: (1) treatment with at least one of: azathioprine, 6-mercaptopurine, methotrexate, corticosteroids, or antibiotics; and (2) none of these treatments. After a $1-2$ week screening period, eligible patients were randomly assigned in a $2: 1$ ratio to CDP571 or placebo. The randomisation schedules for each of the two stratified groups were computer generated by an unblinded statistician at Celltech (Slough, UK).

Disease activity was assessed at the baseline (randomisation) visit, and after 2, 4, 8, 12, 16, 24, and 28 weeks. Patients recorded on diary cards the frequency of loose stools, the extent of their abdominal pain, and their general well being during the seven days before each visit. At each visit, a

Table 1 Baseline characteristics of the patients

\begin{tabular}{|c|c|c|}
\hline Variable & Placebo $(n=132)$ & CDP571 $(n=263)$ \\
\hline White (n (\%)) & $124(93.9)$ & $253(96.2)$ \\
\hline Male (n (\%)) & $43(32.6)$ & $105(39.9)$ \\
\hline Age at entry (y) (mean (SD)) & $37.7(12.5)$ & $37.9(12.4)$ \\
\hline Weight $(\mathrm{kg})$ (mean (SD)) & $68.4(18.7)$ & $70.8(17.4)$ \\
\hline Duration of Crohn's disease (y) (mean (SD)) & $8.4(7.6)$ & $9.1(8.1)$ \\
\hline \multicolumn{3}{|l|}{ Disease site (n (\%)) } \\
\hline lleum & 25 (18.9) & $45(17.1)$ \\
\hline \|leocolon & $71(53.8)$ & $145(55.1)$ \\
\hline Colon & $35(26.5)$ & $72(27.4)$ \\
\hline Other & $1(0.8)$ & $1(0.4)$ \\
\hline \multicolumn{3}{|l|}{ Open and draining perianal or abdominal enterocutaneous } \\
\hline Previous intestinal resection (n (\%)) & $43(32.6)$ & $106(40.3)$ \\
\hline CDAl score (mean (SD)) & $301(61)$ & $297(63)$ \\
\hline IBDQ score (mean (SD)) & $124(28)$ & $129(29)$ \\
\hline CRP concentration $(\mathrm{mg} / \mathrm{l})$ (geometric mean $(95 \% \mathrm{CI})$ ) & $7.5(5.6-10.0)$ & $6.7(5.5-8.0)$ \\
\hline \multicolumn{3}{|l|}{ Concomitant medications (n (\%)) } \\
\hline${ }^{1}$ Corticosteroids (includes budesonide) & $54(40.9)$ & $109(41.4)$ \\
\hline${ }^{2}$ Azathioprine or 6-mercaptopurine & $36(27.3)$ & $81(30.8)$ \\
\hline${ }^{3}$ Methotrexate & $4(3.0)$ & $9(3.4)$ \\
\hline${ }^{4}$ Antibiotics & $18(13.6)$ & $39(14.8)$ \\
\hline${ }^{5} 5-$ Aminosalicylates $^{*}$ & $76(57.6)$ & $157(59.7)$ \\
\hline One or more of 1,2 , or 3 & $77(58.3)$ & $154(58.6)$ \\
\hline One or more of $1,2,3$, or 4 & $82(62.1)$ & $160(60.8)$ \\
\hline Past anti-TNF- $\alpha$ therapy (n (\%)) & $28(21.2)$ & $63(24.0)$ \\
\hline
\end{tabular}




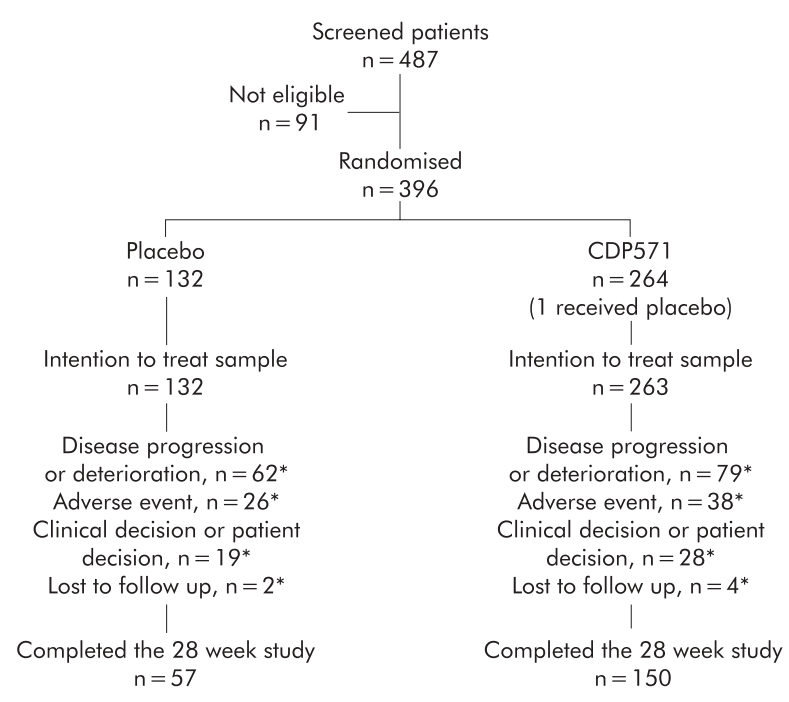

Figure 1 Trial profile. * Indicates that some patients withdrew from the trial for more than one reason. A total of 76 patients in the placebo group and 113 patients in the CDP571 trial withdrew prior to week 28 .

physical examination, fistula evaluation (inspection of the fistula for closure, with closure being defined as no drainage on gentle compression), quality of life assessment, and laboratory tests were conducted and patients were asked whether any adverse events had occurred.

Clinical disease activity was assessed using the CDAI. ${ }^{14}$ Patients were assessed at each visit for the presence of open and actively draining perianal or abdominal enterocutaneous fistulae (defined as open fistulae with either spontaneous drainage or the ability to express drainage with gentle compression). ${ }^{4}$ Disease specific health related quality of life was assessed with the self administered inflammatory bowel disease questionnaire (IBDQ). ${ }^{15} 16$

Blood samples were taken for haematological and biochemical assessments, and for measurement of plasma antiCDP571 antibody (immunogenicity assay) and $\mathrm{C}$ reactive protein (CRP) concentrations.

All adverse events were recorded and graded according to the Medical Dictionary for Regulatory Activities dictionary criteria. Infusion reactions were defined as any adverse events occurring within two hours of infusion of the study medication.

\section{Outcomes and statistical analysis}

The intention to treat (ITT) population included all patients who were evaluated at the screening and baseline visits and who received at least one dose of study medication. The primary efficacy variable was the percentage of patients who showed clinical response (a decrease in CDAI score of $\geqslant 100$ points from baseline or remission (CDAI score $\leqslant 150)$ ) at week 28. The major secondary efficacy variable was the percentage of patients who showed clinical response at week 2. Other secondary efficacy variables included: (1) percentage of patients who showed a clinical response at weeks $4,8,12$, 16 , and $24 ;(2)$ percentage of patients who were in clinical disease remission (CDAI score $\leqslant 150$ ) at weeks 2, 4, 8, 12, 16, 24 , and 28; (3) percentage of patients who showed a decrease in CDAI score of $\geqslant 70$ points at weeks $2,4,8,12,16,24$, and 28; (4) CDAI and IBDQ scores at weeks 2, 4, 8, 12 (CDAI only), 16, 24, and 28; (5) percentage of patients in the subgroup with fistulae who showed closure of at least 50\% and $100 \%$ of fistulae at any visit and on two consecutive visits over a six week period, closure being defined as no drainage on gentle compression; and (6) CRP concentrations at weeks 4, 8, 16, and 28. Safety endpoints were: (1) adverse events; (2) laboratory data; and (3) the presence of antibodies to CDP571 at weeks $0,8,16$, and 24. A post hoc exploratory analysis was performed to test the hypothesis that elevated baseline CRP concentration (CRP $\geqslant 10 \mathrm{mg} / \mathrm{l}$ ) might be a predictor of response to CDP571, as measured by the percentage of patients who showed clinical response. We also performed exploratory comparisons to determine the impact of concomitant therapy with azathioprine or 6-mercaptopurine, concomitant therapy with corticosteroids, and past therapy with infliximab on the percentage of patients who showed clinical response at weeks 2, 4, 8, 12, 16, 24 , and 28.

The percentages of patients who showed a clinical response (decrease in CDAI score of $\geqslant 100$ points or achieved remission $($ CDAI $\leqslant 150)$ ), a decrease in CDAI score of $\geqslant 70$ points (secondary end point), or who were in clinical disease remission (CDAI score $\leqslant 150$ ) at weeks $2,4,8,12,16,24$, and 28 were summarised and compared between treatment groups using the Mantel-Haenszel test, adjusting for steroid and/or immunosuppressant and/or antibiotic use. CDAI scores, IBDQ scores, and CRP concentrations were summarised at weeks 2 (CDAI, IBDQ only), 4, 8, 12 (CDAI only), 16, 24 (CDAI and IBDQ only), and 28, compared with baseline values, and presented graphically. Of the subgroup of patients with fistulae, the percentage showing closure of at

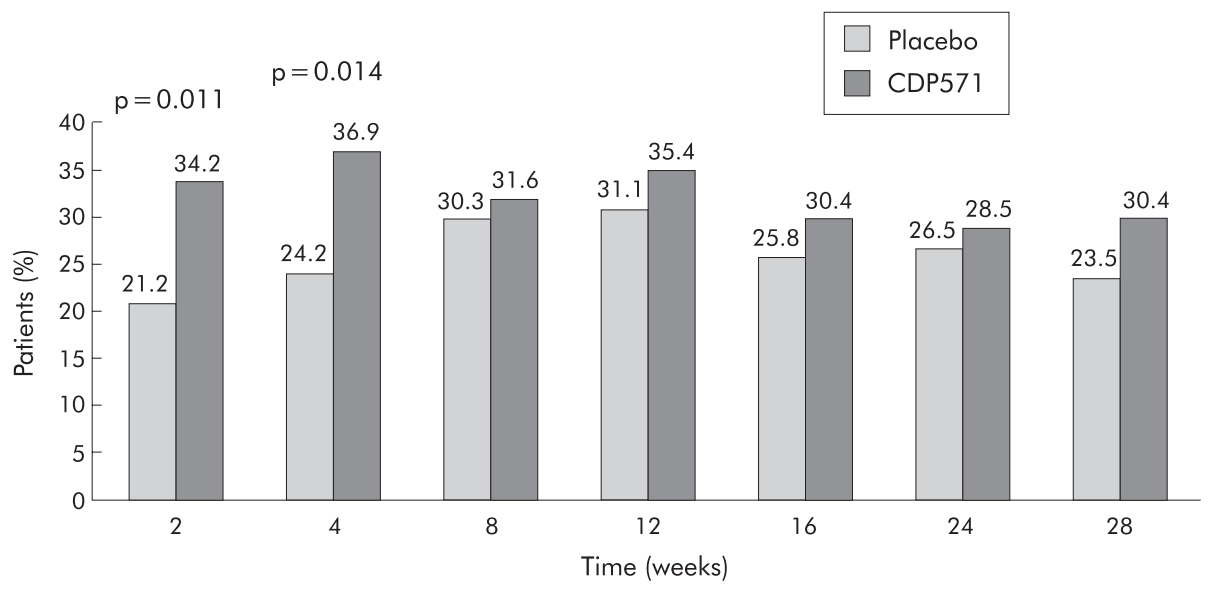

Figure 2 Percentages of patients with Crohn's disease at each study visit who experienced a clinical response (decrease in Crohn's disease activity index (CDAl) score $>100$ points or who achieved clinical remission (CDAl score $\leqslant 150$ points)) in the placebo and CDP571 groups (ITT population). 


\begin{tabular}{|c|c|c|c|c|}
\hline Variable & $\begin{array}{l}\text { Time point } \\
\text { (week) }\end{array}$ & $\begin{array}{l}\text { Placebo } \\
(n=132)\end{array}$ & $\begin{array}{l}\text { CDP571 } \\
(n=263)\end{array}$ & p Value \\
\hline \multirow{7}{*}{$\leqslant 150$ points) } & 2 & $16(12.1)$ & $40(15.2)$ & 0.498 \\
\hline & 4 & $21(15.9)$ & $54(20.5)$ & 0.300 \\
\hline & 8 & $30(22.7)$ & $50(19.0)$ & 0.388 \\
\hline & 12 & $33(25.0)$ & $66(25.1)$ & 0.982 \\
\hline & 16 & $25(18.9)$ & $61(23.2)$ & 0.283 \\
\hline & 24 & $28(21.2)$ & $60(22.8)$ & 0.666 \\
\hline & 28 & $27(20.5)$ & $62(23.6)$ & 0.429 \\
\hline \multirow[t]{7}{*}{ Decrease in CDAl score of $\geqslant 70$ points } & 2 & $33(25.0)$ & $117(44.5)$ & 0.000 \\
\hline & 4 & $43(32.6)$ & $110(41.8)$ & 0.095 \\
\hline & 8 & $52(39.4)$ & $97(36.9)$ & 0.577 \\
\hline & 12 & $49(37.1)$ & $113(43.0)$ & 0.249 \\
\hline & 16 & $39(29.5)$ & $95(36.1)$ & 0.201 \\
\hline & 24 & $47(35.6)$ & $91(34.6)$ & 0.795 \\
\hline & 28 & $36(27.3)$ & $95(36.1)$ & 0.072 \\
\hline Closure of at least $50 \%$ of fistulae & At any visit & $9(34.6)^{*}$ & 33 (55.0)† & 0.103 \\
\hline Closure of $100 \%$ of fistulae & At any visit & $8(30.8)^{*}$ & $26(43.3) \dagger$ & 0.340 \\
\hline Closure of at least $50 \%$ of fistulae & $\begin{array}{l}\text { At any } 2 \\
\text { consecutive visits } \\
\text { over a } 6 \text { week } \\
\text { period }\end{array}$ & $5(19.2)^{*}$ & $16(26.7) \dagger$ & 0.589 \\
\hline Closure of $100 \%$ of fistulae & $\begin{array}{l}\text { At any } 2 \\
\text { consecutive visits } \\
\text { over a } 6 \text { week } \\
\text { period }\end{array}$ & $5(19.2)^{*}$ & $15(25.0) \dagger$ & 0.782 \\
\hline
\end{tabular}

least $50 \%$ and $100 \%$ of fistulae at any visit and on two consecutive visits over a six week period at any time during the study were summarised and comparisons between treatment groups were made using Fisher's exact test. Adverse events were summarised using descriptive statistical techniques by body system, preferred term, and severity, as well as by body system, preferred term, and relationship to study drug. The percentages of patients with antibodies to CDP571 were also descriptively summarised. Post hoc exploratory comparisons to determine the impact of an elevated baseline CRP concentration $(\geqslant 10 \mathrm{mg} / \mathrm{l})$, concomitant therapy with azathioprine or 6-mercaptopurine, concomitant therapy with corticosteroids, and antecedent therapy with infliximab on the percentage of patients who showed clinical response at weeks 2, 4, 8, 12, 16, 24, and 28 were performed with Fisher's exact test. For analyses of CDAI scores, IBDQ scores, and CRP values, data for patients who were lost to follow up or who withdrew from the study because of deterioration in their condition or adverse events were censored at the time of the last study visit (a last value carried forward approach was not used for these analyses). All statistical tests were two sided and $p$ values $<0.05$ were considered statistically significant.

\section{Sample size}

We estimated that 256 patients were needed in the CDP571 group and 128 patients in the placebo group in order to have $90 \%$ power to detect a true difference in the proportion of patients who achieved clinical response (reduced their CDAI score by $\geqslant 100$ points or achieved remission (CDAI score $\leqslant 150)$ ) at week 28 , assuming a true difference of $12.5 \%$ and a placebo rate of $7 \%$. We planned to recruit a total of 384 patients.

\section{RESULTS}

Four hundred and eighty seven patients were screened, of whom 396 were randomised. The number of patients randomised in each country was as follows: Australia 85 (21.5\%), Bulgaria 14 (3.5\%), Canada 44 (11.1\%), Czech
Republic 28 (7.1\%), Hungary 42 (10.6\%), Poland 38 (9.6\%), and the USA $145(36.6 \%)$. Of the 396 randomised patients, 133 received placebo and 263 received CDP571. One patient was randomised to receive CDP571 but actually received placebo; this patient was included in the placebo group for the safety population and in the CDP571 group for the other analyses. No post baseline efficacy data were obtained for one of the patients and the remaining 395 patients comprised the ITT population. Baseline characteristics of the two groups of patients were similar (table 1). The disposition of participating patients is shown in fig 1 .

\section{Clinical effectiveness}

The percentage of patients who had a clinical response (decrease in CDAI score of $\geqslant 100$ points or remission (CDAI score $\leqslant 150$ points)) at week 28 (primary end point) was not significantly higher in patients treated with CDP571 (80/263; $30.4 \%)$ than in those who received placebo $(31 / 132,23.5 \%$; $\mathrm{p}=0.102$ ). However, at week 2 (major secondary end point) and week 4 (secondary end point) there were significant differences in clinical response rates in favour of CDP571 (fig 2). At week 2, clinical response occurred in 90/263 $(34.2 \%)$ CDP571 patients and 28/132 (21.2\%) placebo patients $(p=0.011)$. There were no differences in clinical response between the treatment groups at weeks 8, 12, 16, and 24. The percentages of patients who achieved clinical remission (CDAI score $\leqslant 150$ ) at weeks 2, 4, 8, 12, 16, 24, and 28 (secondary end points) were similar in the two treatment groups (table 2). The percentages of patients who achieved a decrease in CDAI score of $\geqslant 70$ points at weeks 2 and 4 (secondary end points) were significantly higher in patients treated with CDP571 compared with patients treated with placebo but there were no significant differences at weeks 8 , $12,16,24$, and 28 (secondary end points) (table 2). Mean CDAI scores at weeks 2, 4, 8, 12, 16, 24, and 28 (secondary end points) were lower in patients treated with CDP571 but these differences were not significant (fig 3A). The mean IBDQ scores at weeks 2, 4, 8, 16, 24, and 28 (secondary end points) were higher in patients treated with CDP571 but 

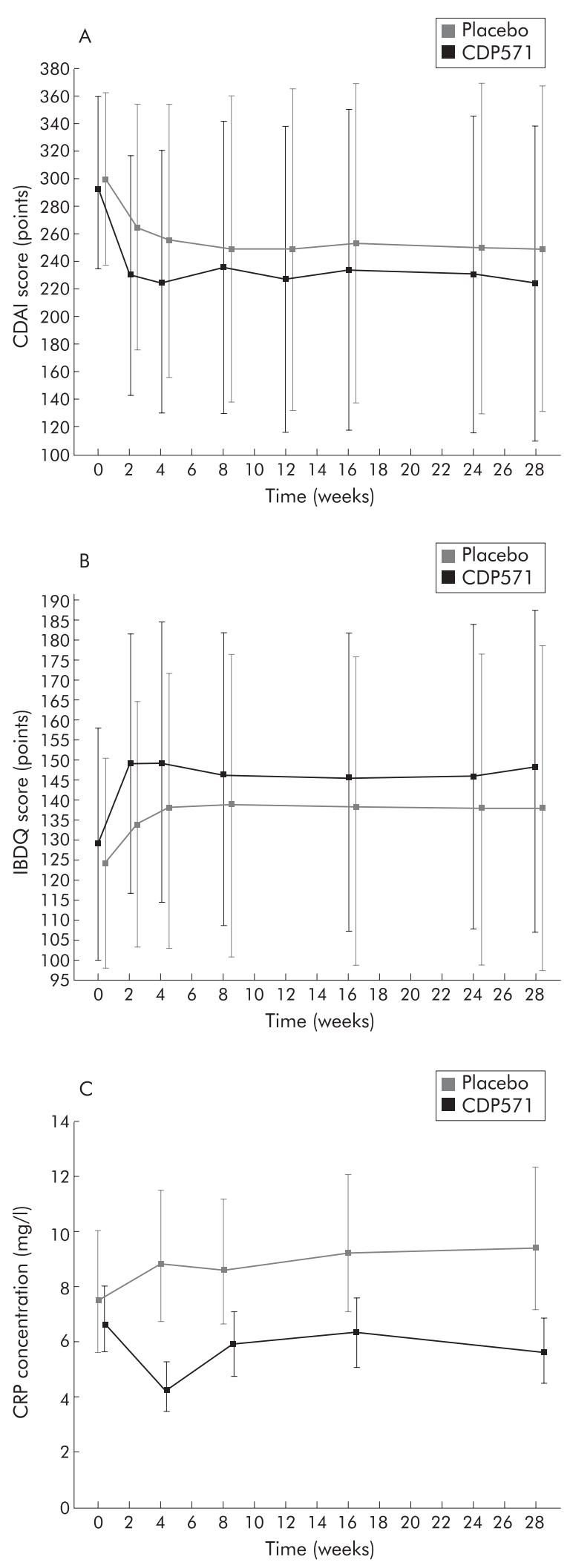

Figure 3 Mean scores or values at each study visit, according to treatment group. (A) Crohn's disease activity index (CDAl) scores; (B) inflammatory bowel disease questionnaire (IBDQ) scores; and (C) C reactive protein (CRP) values (geometric means). Error bars represent SD values $(A, B)$ or $95 \%$ confidence intervals $(C)$.

these differences were not significant (fig 3B). The percentages of patients who had closure of at least $50 \%$ of fistulae at any visit, closure of at least $50 \%$ of fistulae for any two consecutive visits over six weeks, closure of $100 \%$ of fistulae at any visit, and closure of $100 \%$ of fistula for any two consecutive visits over six weeks (secondary end points) were similar in the two treatment groups (table 2). Based on the confidence intervals, the geometric mean CRP concentrations at weeks 4 and 28 (secondary end points) were lower in patients treated with CDP571 than in placebo treated patients (fig 3C).

Exploratory comparisons were made to determine the impact of an elevated baseline CRP concentration ( $\geqslant 10 \mathrm{mg} / \mathrm{l})$, concomitant therapy with azathioprine or 6-mercaptopurine, concomitant therapy with corticosteroids, and antecedent therapy with infliximab on the percentage of patients who showed a clinical response (decrease in CDAI score of $\geqslant 100$ points or who achieved remission $($ CDAI $\leqslant 150)$ ) at weeks 2, 4, 8, 12, 16, 24, and 28. Concomitant therapy with azathioprine or 6-mercaptopurine, concomitant therapy with corticosteroids, and antecedent therapy with infliximab did not influence the percentage of patients who showed a clinical response at weeks $2,4,8,12,16,24$, and 28 (data not shown). There were no significant differences in the outcomes for any of the primary or secondary end points according to the stratification criteria (concomitant therapy with at least one of: corticosteroids, azathioprine, 6-mercaptopurine, methotrexate, or antibiotics). Likewise, multivariate analyses identified no interactions between the treatment response and any of the baseline characteristics of the patients.

One hundred and fifty nine of 395 (40.3\%) patients had an elevated baseline CRP concentration $\geqslant 10 \mathrm{mg} / \mathrm{l}$. We observed that patients with an elevated baseline CRP concentration who received CDP571 were more likely to show a clinical response (decrease in CDAI score of $\geqslant 100$ points or who achieved clinical remission (CDAI score $\leqslant 150)$ ) at weeks 2 , $12,16,24$, and 28 than those who received placebo (fig 4A). A trend towards significance was observed for the comparison at week 4. Conversely, no such difference was observed for patients with a baseline CRP concentration $<10 \mathrm{mg} / \mathrm{l}$ (fig 4B).

\section{Adverse events}

The two treatment groups were similar with regard to the percentages of patients with: any adverse event; serious adverse events; adverse events leading to withdrawal from the study; severe adverse events; adverse events probably related to the study medication; and adverse events definitely related to the study medication (table 3 ). The most frequent (75\%) adverse events were generally similar between the two treatment groups (table 3 ). The percentage of patients with infusion reactions (defined as adverse events occurring within two hours of the start of the study medication infusion) was greater in patients treated with CDP571 $(20.5 \%)$ compared with placebo $(10.5 \%)$ (table 3$)$. The percentage of patients with infections was the same in the two treatment groups (29.3\%) (table 3). One patient in the placebo group developed adenocarcinoma, with the rectum being the most likely primary site. One patient in the CDP571 group developed cervical cancer. This was discovered incidentally (CIN stage II) and was considered to be unrelated to the study drug. No patient developed tuberculosis or opportunistic infections, and no patient died from any cause in either treatment group during the study. The frequency of anti-idiotype antibodies to CDP571 was $(10.9 \%) 27 / 247$ in the CDP571 treatment group. The frequency of these antibodies was (4.7\%) 4/86 in patients treated with CDP571 who received concomitant antimetabolite therapy compared with $(14.3 \%) 23 / 161$ in those who did not receive these drugs. There were no clinically significant changes in laboratory values in either treatment group. 

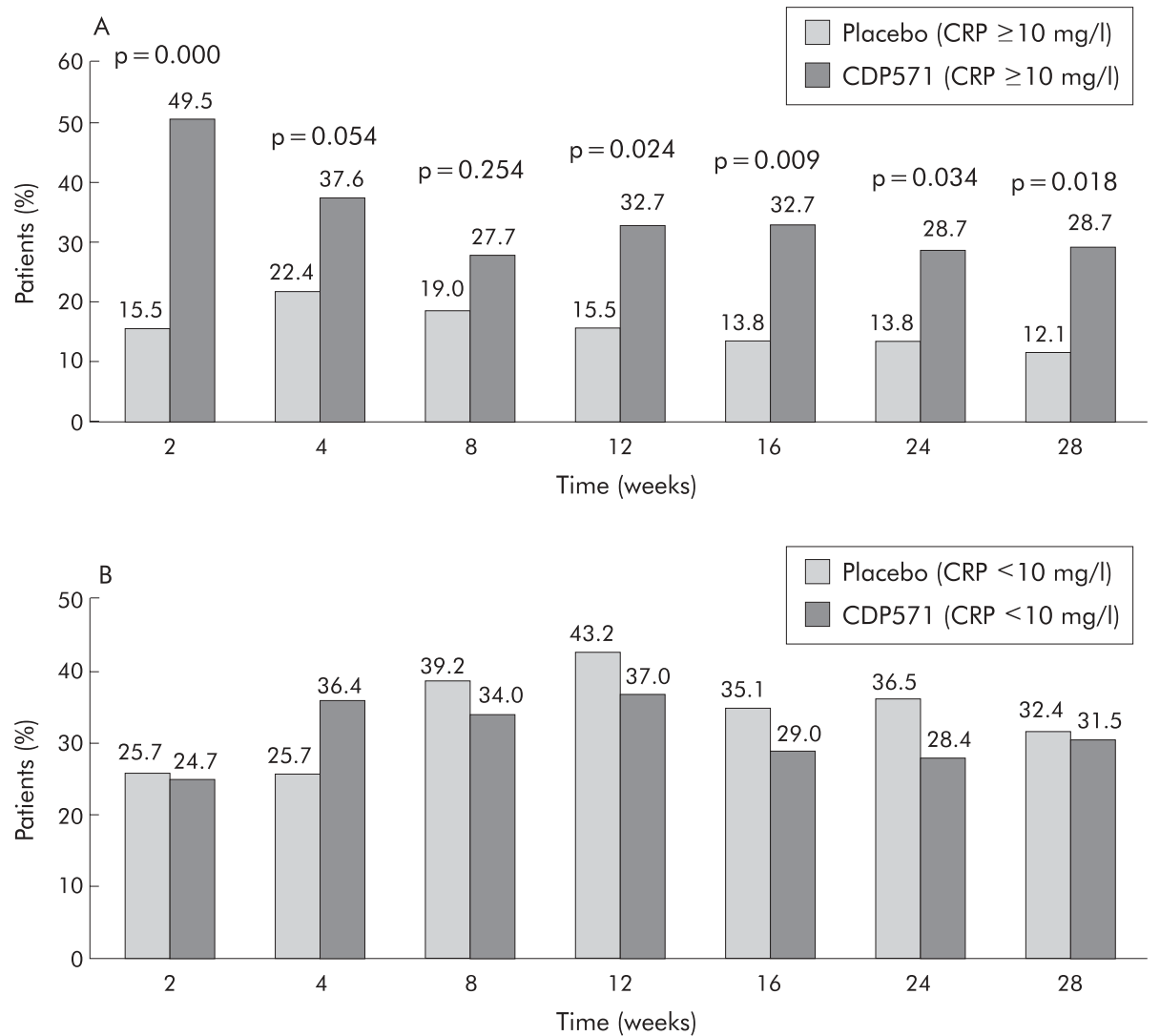

Figure 4 Percentages of patients with Crohn's disease at each study visit who experienced a clinical response (decrease in Crohn's disease activity index (CDAl) score $>100$ points or who achieved clinical remission (CDAl score $\leqslant 150$ points)) according to baseline $C$ reactive protein (CRP) concentration. (A) Patients with a baseline CRP concentration $\geqslant 10 \mathrm{mg} / \mathrm{l}$; (B) patients with a baseline CRP $<10 \mathrm{mg} / \mathrm{l}$.

Table 3 Adverse events in the two treatment groups

\begin{tabular}{lcc}
\hline & \multicolumn{2}{l}{ No of patients (\%) } \\
\cline { 2 - 3 } & CDP571 & Placebo \\
(n=263)
\end{tabular}




\section{DISCUSSION}

We found CDP571 to be effective for inducing a short term clinical response at weeks 2 and 4 (secondary end points) in unselected patients with moderate to severe Crohn's disease. However, the clinical relevance of this short term response, which was not maintained through to week 28 (primary end point), is unclear. This lack of sustained efficacy in the overall study patient population was confirmed by failure to achieve statistical significance for most other secondary end points. Thus although CDP571 can induce a modest short term clinical response, it does not appear to be suitable for the long term treatment of unselected patients with moderate to severe Crohn's disease. It should however be borne in mind that this study was concerned with acute treatment of patients with Crohn's disease and a primary end point based on the results at week 28 may not be the most appropriate end point in such an instance.

Post hoc exploratory analysis was performed to determine whether patients with elevated baseline CRP concentrations had greater rates of clinical response than the overall patient population. Among these selected patients with elevated baseline CRP ( $\geqslant 10 \mathrm{mg} / \mathrm{l})$, the rates of clinical response in CDP571 treated patients were significantly greater than in the placebo treated group at weeks 2, 12, 16, 24, and 28 (with a trend towards statistical significance at week 4). There were no significant differences in clinical response rates between the treatment groups throughout the 28 week study for patients in whom the baseline CRP concentration was $<10 \mathrm{mg} / \mathrm{l}$. Interestingly, a feature of this analysis is the lower placebo response in patients with $\mathrm{CRP} \geqslant 10 \mathrm{mg} / \mathrm{l}$ compared with those in whom baseline concentrations of this protein were not elevated. It is possible that patients with higher levels of CRP may be less likely to exhibit spontaneous improvement.

Analysis of the adverse events associated with the administration of CDP571 showed that it was well tolerated. Indeed, combining the results of the trial described here with five other published studies ${ }^{11-13} 17^{18}$ gives a total of 960 patients with Crohn's disease who have been treated with either CDP571 or placebo. The combined safety data from these six studies, as well as those from two open label extension studies, shows CDP571 to have a favourable safety profile similar to the results of this study (Celltech, Slough, UK, data on file). In addition, CDP571 appears to have a relatively low immunogenicity profile. The frequency of antiidiotype antibodies to CDP751 (10.9\%) compares favourably with results that have been reported for infliximab, where as many as $60 \%$ of retreated patients have developed antibodies. $^{367}$ These contrasting results demonstrate the benefits of using a "humanised" anti-TNF- $\alpha$ antibody.

In conclusion, CDP571 is modestly effective for short but not long term treatment of unselected patients with moderate to severe Crohn's disease. The clinical relevance of this short term effect is unclear. Subgroup analysis suggests both short and long term efficacy of CDP571 in selected patients with elevated baseline CRP $(\geqslant 10 \mathrm{mg} / \mathrm{l})$. CDP571 is well tolerated.

\section{ACKNOWLEDGEMENT}

Supported by a research grant from Celltech, Slough, UK.

\author{
Authors' affiliations \\ W J Sandborn, Mayo Clinic, Rochester, Minnesota, USA \\ B G Feagan, University of Western Ontario, London, Ontario, Canada \\ G Radford-Smith, Royal Brisbane Hospital, Herston, Queensland, \\ Australia \\ A Kovacs, Peterfy Hospital, Budapest, Hungary
}

R Enns, St Paul's Hospital, University of British Colombia, Vancouver, British Columbia, Canada

A Innes, J Patel, Celltech Research and Development, Slough, UK

Conflicts of interest: W Sandborn and B Feagan have served as consultants for Celltech. A Innes is an employee of Celltech. J Patel is a former employee of Celltech.

\section{APPENDIX}

\section{INVESTIGATORS}

\section{Australia}

1. Brett Jones, Division of Medicine, the Liverpool Hospital, Liverpool, NSW, Australia

2. Peter Gibson, Department of Medicine, Royal Melbourne Hospital, Parkville, Victoria, Australia

3. Bill Connell, St Vincent's Hospital Melbourne, Fitzroy, Victoria, Australia

4. Graham Radford-Smith, Department of Gastroenterology, Royal Brisbane Hospital, Herston, Queensland, Australia

5. Timothy Florin, Mater Miser Hospital, South Brisbane, Queensland, Australia

6. Peter Bampton, Flinders Medical Center, Bedford Park, South Australia, Australia

7. Stephen Riordan, Prince of Wales Hospital, Randwick, New South Wales, Australia

8. Doug Taupin, the Canberra Hospital, Garran, Australian Capital Territory, Australia

9. Andrew Hallam, Peninsula Specialist Centre, KippaRing, Queensland, Australia

10. John Masson, Department of Endoscopy, Townsville General Hospital, Townsville, Australia

11. Ian Lawrance, Fremantle Hospital, Fremantle, Western Australia, Australia

12. Grant Phelps, Ballarat Gastoenterology, Ballarat, Victoria, Australia

13. Peter Katelaris, Concord Repatriation Hospital, Concord, New South Wales, Australia

\section{Bulgaria}

1. Z Krastev, Clinic of Gastroenterology, Medical UniversitySofia, Sofia, Bulgaria

2. S Stoynov, DUB "Tzaritza Ioana" Sofia, Bulgaria

\section{Canada}

1. Jeffrey Baker, St Michael's Hospital, Toronto, ON, Canada

2. Charles Bernstein, Health Sciences Centre, Winnipeg, MB, Canada

3. Laurington DaCosta, Hotel-Dieu Hospital, Kingston, ON, Canada

4. Robert Enns, St Paul's Hospital, Vancouver, BC, Canada

5. Brian Feagan and James Gregor, London Health Sciences Centre, London, ON, Canada

6. David A Lloyd, St Joseph's Health Care London, London, ON, Canada

7. Lloyd Sutherland, University of Calgary, Health Science Centre, Calgary, AB, Canada

\section{Czech Republic}

1. Petr Zdenek, lst Internal Clinic-Gastroenterology, Faculty Hospital, Lochotin, Czech Republic 
2. Josef Hajek, Internal Clinic B, Internal Department 3, Pardubice, Czech Republic

3. Miroslava Volfova, Faculty Hospital Hradec Králové, Hradec Králové, Czech Republic

4. Ivan Gregar, Faculty Hospital-Internal Clinic, Olomouc, Czech Republic

5. Milan Lukas, IV Internal Clinic Gastroenterology, General Faculty Hospital, Prague, Czech Republic

6. Jiri Stehlik, Gastroenterology, Internal Clinic, Masaryk's Hospital, Ústí nad Lbem, Czech Republic

7. Miroslav Zavoral, Central Military Hospital, Iind Department of Internal Medecine, Prague, Czech Republic

8. Libor Gabalec, District Hospital Usti nad Orlici, Department of Internal Medecine, Usti nad Orlici, Czech Republic

\section{Hungary}

1. Miklos Udvardy, University Clinic (Debrecen), Debrecen, Hungary

2. Tamas Zagoni, II Medical Department, Semmelweis University, Szentkiralyi, Budapest, Hungary

3. Janos Lonovics, I Medical Department, Szent Gyorgyi Albert University, Szeged, Hungary

4. Gyorgy Nagy, II Medical Department, BAZ County Hospital, Miskolc, Hungary

5. Laszlo Lakatos, I Medical Department, Veszprem County Hospital, Veszprem, Hungary

6. Agota Kovacs, Peterfy Hospital, I Internal Medicine Department, Budapest, Hungary

\section{Poland}

1. Krzysztof Marlicz, Deptartment of Gastroenterology, Pomeranian Medical Academy, Szczecin, Poland

2. Jan Dzieniszewski, Gastroenterology Clinic, Brodnowski Hospital, Warsaw, Poland

3. Grazyna Rydzewska, Gastroenterology Clinic, Central Clinical Hospital of Ministry of Internal Affairs, Warsaw, Poland

4. Leszek Paradowski, Deptartment of Gastroenterology, Medical Academy, Wroclaw, Poland

5. Krzysztof Linke, Internal Diseases Institute, Poznan, Poland

6. Józef Bogdal, Department of Gastroenterology, Collegium Medicum of the Jagiellonian University, Cracow, Poland

7. Andrzej Kryszewski, Institute of Internal Medicine, Medical Academy of Gdansk, Gdansk, Poland

8. Professor Krasnodebski, Surgery Gastroenterology Deptartment, Warsaw, Poland

\section{USA}

1. Stephen J Bickston, University of Virginia Health System, Charlottesville, VA, USA

2. Jeffrey Breiter, Center for Medical Research LLC, Manchester, CT, USA

3. Florian Cortese, Mercury Street Medical Group, Butte, MT, USA

4. Lance DeFrancisco, Altoona, PA, USA

5. Willem de Villiers, Internal Medicine and GI, UK Chandler Medical Center, Lexington, KY, USA
6. Richard J Elkin, Regional Gastroenterology Associates of Lancaster, Lancaster, PA, USA

7. David Eskreis and Seymour Katz, Long Island Clinical Research Association, Great Neck, NY, USA

8. John S Goff, Rocky Mountain Clinical Research, Lakewood, CO, USA

9. Stanley Goldberg, Gastroenterology Association of the East Bay Medical Group, Berkeley, CA, USA

10. Mark Griffin, Gastroenterology Specialties, Lincoln, NE, USA

11. Stephen B Hanauer, University of Chicago, Chicago, IL, USA

12. Robert Hardi, Chevy Chase Clinical Research, Chevy Chase, MD, USA

13. William R Harlan, Asheville Gastroenterology Association, Asheville, NC, USA

14. Gary R Lichtenstein, University of Pennsylvania Hospital, Philadelphia, PA, USA

15. Donald C Lipkis, Institute of Healthcare Assessment, San Diego, CA, USA

16. Gregory Mula, New Orleans Clinical Trial Management, Covington, LA, USA

17. Frederick Opper, Hanover Med Spec PA, Wilmington, NC, USA

18. Daniel Pambianco, Charlottesville Medical Research, Charlottesville, VA, USA

19. William Priebe, Tacoma Digestive Disease Research, Tacoma, WA, USA

20. Ron Pruitt, Nashville Medical Research Institute, Nashville, TN, USA

21. Michael Safdi, Consultants for Clinical Research, Cincinnati, OH, USA

22. William J Sandborn, Edward V Loftus, and William J Tremaine, Mayo Clinic, Rochester, MN, USA

23. David Stanton, Community Clinical Trials, Orange, CA, USA

24. Douglas Wolf, Atlanta Gastroenterology Associates, Atlanta, GA, USA

\section{REFERENCES}

1 Targan SR, Hanauer SB, van Deventer SJ, et al. A short-term study of chimeric monoclonal antibody cA2 to tumor necrosis factor $\alpha$ for Crohn's disease. Crohn's Disease cA2 Study Group. N Engl J Med 1997;337:1029-35.

2 Rutgeerts P, D'Haens G, Targan S, et al. Efficacy and safety of retreatment with anti-tumor necrosis factor antibody (infliximab) to maintain remission in Crohn's disease. Gastroenterology 1999;117:761-9.

3 Hanaver SB, Feagan BG, Lichtenstein GR, et al. Maintenance infliximab for Crohn's disease: the ACCENT I randomised trial. Lancet 2002;359:1541-9.

4 Present DH, Rutgeerts $\mathrm{P}$, Targan $\mathrm{S}$, et al. Infliximab for the treatment of fistulas in patients with Crohn's disease. N Engl J Med 1999;340:1398-405.

5 Sands B, Van Deventer S, Bernstein C, et al. Long-term treament of fistulizing Crohn's disease: response to infliximab in the ACCENT II trial through 54 weeks. Gastroenterology 2002;122:A81.

6 Baert F, Noman M, Vermeire S, et al. Influence of immunogenicity on the longterm efficacy of infliximab in Crohn's disease. N Engl J Med 2003;348:601-8.

7 Farrell RJ, Alsahli M, Jeen YTJ, et al. Intravenous hydrocortisone premedication to prevent antibodies to infliximab in Crohn's disease: a randomized controlled trial. Gastroenterology 2003;124:917-24.

8 Hanauer S, Rutgeerts $P$, Targan S, et al. Delayed hypersensitivity to infliximab (Remicade) re-infusion after a 2-4 year interval without treatment. Gastroenterology 1999;116:A731.

9 Winter G, Harris WJ. Humanized antibodies. Immunol Today 1993;14:243-6.

10 Stephens S, Emtage S, Vetterlein O, et al. Comprehensive pharmacokinetics of a humanized antibody and analysis of residual anti-idiotypic responses. Immunology 1995;85:668-74. 
11 Stack WA, Mann SD, Roy AJ, et al. Randomised controlled trial of CDP571 antibody to tumour necrosis factor- $\alpha$ in Crohn's disease. Lancet 1997;349:521-4.

12 Sandborn WJ, Feagan BG, Hanauer SB, et al. An engineered human antibody to TNF (CDP571) for active Crohn's disease: a randomized doubleblind placebo-controlled trial. Gastroenterology 2001;120:1330-8.

13 Feagan BG, Sandborn WJ, Baker J, et al. A randomized, double-blind, placebo-controlled, multi-center trial of the engineered human antibody to TNF (CDP571) for steroid sparing and maintenance of remission in patients with steroid-dependent Crohn's disease. Gastroenterology 2000:118:A655.

14 Best WR, Becktel JM, Singleton JW, et al. Development of a Crohn's disease activity index. National Cooperative Crohn's Disease Study. Gastroenterology 1976;70:439-44
15 Irvine EJ, Feagan B, Rochon J, et al. Quality of life: a valid and reliable measure of therapeutic efficacy in the treatment of inflammatory bowel disease. Canadian Crohn's Relapse Prevention Trial Study Group. Gastroenterology 1994;106:287-96.

16 Irvine EJ Feagan BG, Wong CJ. Does self-administration of a quality of life index for inflammatory bowel disease change the results? J Clin Epidemiol 1996:49: 1177-85.

17 Celltech announces results from CDP571 Phase III studies in Crohn's disease. Internet Press Release, 2002.

18 Hanquer S, Present D, Targan SR, et al CDP571, a humanized monoclonal antibody to TNF- $\alpha$, is well tolerated in Crohn's disease patients with previous hypersensitivity to infliximab. Digestive Disease Week, Orlando, FL, USA 2003: abstract T, 1357

\title{
EDITOR'S QUIZ: GI SNAPSHOT
}

\author{
Large abdominal mass in Crohn's disease
}

\section{Clinical presentation}

A 49 year old male patient with a 23 year history of Crohn's disease was admitted to our hospital for the appearance of an abdominal mass in the right upper quadrant of the abdomen. He reported the occurrence of abdominal distension and fullness for two weeks. Two years before he underwent ileocolic resection and six months later surgical treatment of a perianal localisation of his Crohn's disease.

At admission, a large abdominal mass was found on physical examination. Pain was absent. Routine blood examination did not reveal abnormal values. Tumoral marker levels, including CEA and CA 19.9, were negative.

An abdominal ultrasound revealed a large $(13 \mathrm{~cm}$ in diameter) right upper quadrant mass. A computerised tomography scan showed a bulky abdominal neoplasm, with sharp tumour margins and homogeneous density before and after intravenous contrast delivery.

\section{Question}

A small bowel radiography was also performed (fig 1). What is the diagnosis?

See page 1503 for answer

This case is submitted by:

M Caricato, F Ausania, D Borzomati, S Valeri, R Coppola Department of Surgery, Campus Bio-Medico University, Rome, Italy

A Verzi

Department of Surgical Pathology, Campus Bio-Medico University, Rome,

Italy

G Tonini

Department of Oncology, Campus Bio-Medico University, Rome, Italy

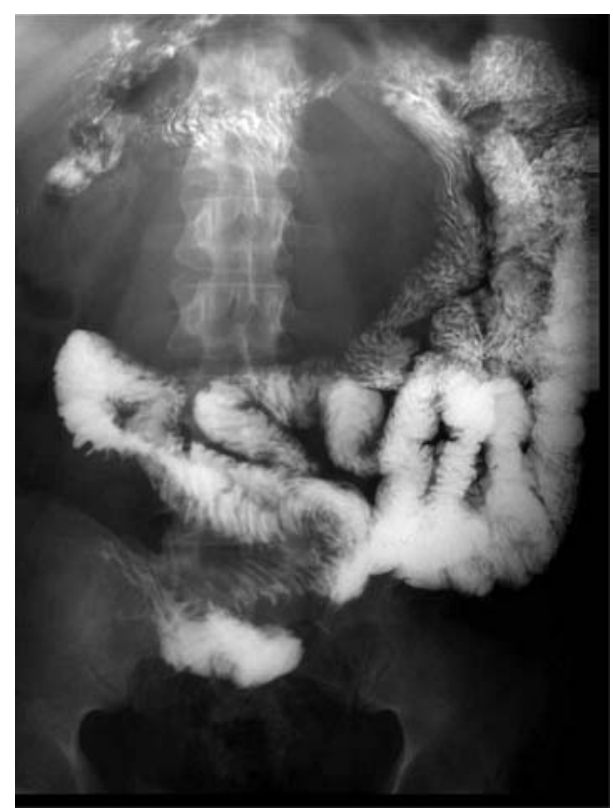

Figure 1 Small bowel radiography.

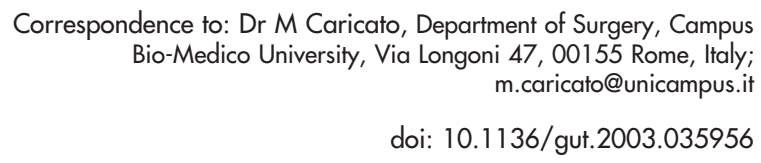

